\title{
Diagnosis and management of acute kidney injury: deficiencies in the knowledge base of non-specialist, trainee medical staff
}

\author{
TM Muniraju, MH Lillicrap, JL Horrocks, JM Fisher, RMW Clark and NS Kanagasundaram
}

\begin{abstract}
Enhanced education has been recommended to improve non-specialist management of acute kidney injury (AKI). However, the extent of any gaps in knowledge has yet to be defined fully. The aim of this study was to assess understanding of trainee doctors in the prevention, diagnosis and initial management of AKI. An anonymised questionnaire was completed by hospital-based trainees across Newcastle Renal Unit's catchment area. Responses were evaluated against a panel of pre-defined ideal answers. The median score was 9.5 out of 20 ( $n=146$; range $0-17)$ and was lower in more junior trainees. Fifty percent of trainees could not define AKI, 30\% could not name more than two risk factors for AKI and $37 \%$ could not name even one indication for renal referral. These serious gaps in knowledge highlight the need for enhanced education aimed at all training grades. Organisational changes may also be required to optimise patient safety.
\end{abstract}

KEY WORDS: acute kidney injury, education, medical, postgraduate, undergraduate

\section{Background}

Acute kidney injury (AKI) is common, ${ }^{1}$ dangerous ${ }^{2-6}$ and expensive. $^{7}$ Survivors of the initial episode may have higher long-term mortality ${ }^{8-10}$ and persistent - and potentially progressive - renal dysfunction. ${ }^{6,9,10}$ However, AKI is potentially preventable in those at risk ${ }^{11}$ - for instance, by avoidance of nephrotoxins and careful fluid management. In those who actually develop AKI, the severity of disease and its sequelae may be mitigated by early recognition and prompt institution of remedial measures.

Unfortunately, practice remains short of the ideal. A number of publications have described deficiencies in both clinical ${ }^{12,13}$ and organisational ${ }^{13-15}$ management in the UK alone. The most comprehensive of these assessments was undertaken by the National Confidential Enquiry into Patient Outcome and Death $(\mathrm{NCEPOD})^{13}$ - an independent body that aims to optimise patient care through research and surveys of practice. One of a

TM Muniraju, ${ }^{1}$ specialist registrar in nephrology; MH Lillicrap, ${ }^{1}$ core trainee; JL Horrocks, ${ }^{2}$ core trainee; JM Fisher, ${ }^{3}$ core trainee; RMW Clark, ${ }^{4}$ core trainee; NS Kanagasundaram, ${ }^{1,5}$ consultant nephrologist and honorary clinical senior lecturer

${ }^{1}$ Renal Services, Newcastle upon Tyne Hospitals NHS Foundation Trust; ${ }^{2}$ Wansbeck General Hospital, Ashington; ${ }^{3}$ North Tyneside General Hospital; ${ }^{4}$ Queen Elizabeth Hospital, Gateshead; ${ }^{5}$ Institute of Cellular Medicine, Newcastle University number of recommendations from NCEPOD was the need to enhance understanding of AKI among non-specialists. However, the extent of any gaps in knowledge has not been fully defined. With this in mind, we assessed the knowledge base of nonspecialist, trainee medical staff within the catchment area of our regional renal unit.

\section{Methods}

The survey was performed between three and six months after publication of the NCEPOD report in June 2009. An anonymised questionnaire was distributed to trainee medical staff at educational meetings in the three base hospitals directly served by the regional renal unit and the three other acute hospitals within its catchment area. The following key topics were assessed: definition, categorisation, risk factors, examination, bedside observations and indications for renal referral.

The purpose of the questionnaire was explained to attendees by one of the co-authors, with reference to NCEPOD's findings. ${ }^{13}$ The change in nomenclature from acute renal failure to AKI was clarified at this time. Completion of the questionnaires was voluntary and took about 10 minutes. Responses were evaluated against a panel of pre-defined ideal answers (Table 1), with a maximum score of 20. The definition of AKI was according to the current staging system of the Acute Kidney Injury Network (AKIN). ${ }^{16}$ Other ideal responses were those that would be expected from non-specialist trainees ${ }^{11}$ and according to current best practice. ${ }^{17}$

Responses were collated and described according to:

- grade of trainee:

- foundation - those within two years after qualification

- specialty - those with at least two years of postgraduate experience; these were further defined as trainee physicians or surgeons

- venue

- trust - the three acute hospitals served directly by the regional renal unit and within the same organisation

- catchment - the three acute hospitals with no direct affiliation to the renal unit but from where AKI referrals were received.

Two-tailed, unpaired t-tests were used to compare questionnaire scores between different groups. Chi-squared tests were used to compare educational experience between different groups. A p value less than 0.05 was deemed to be significant. 


\section{Table 1. Questions with predefined ideal answers and scores.}

\section{Question}

Define AKI

Categorise AKI

Name five key risk factors for developing AKI

Name three aspects of examination that you think are important in assessing AKI

Name three key observations you will ask the bedside nurse to perform in a patient with AKI

\section{Predefined ideal answers}

Maximum score

One point each for:

- Abrupt (within 48 hours) reduction in kidney function

- Serum creatinine increase of $\geqslant 25 \mu \mathrm{mol} / \mathrm{l}$ or increased to $\geqslant 150$ $200 \%$ of baseline

- Urine output $<0.5 \mathrm{ml} / \mathrm{kg} / \mathrm{hour}$ for $>6$ hours

One point each for:

- Pre-renal AKI

- Intrinsic renal AKI

- Post-renal AKI

One point each for:

- Known CKD

- Diabetes mellitus

- Age $>60$ years

- Nephrotoxic medication

- Sepsis

- Hypovolaemia

- Cardiac failure

- Liver disease

One point each for:

- Postural BP

- JVP

- Auscultation of lung bases

- Assessment of fluid status

- Urinalysis

- Auscultation for a third heart sound

- Examination for peripheral oedema

- Systemic manifestations, eg rash

- Palpation for a full bladder

- Weight in comparison to premorbid measurement

- Other - retrospectively determined by co-author consensus

One point each for:

- MEWS score

- Fluid balance charting

- Daily weights

- Postural BP

Half point each for:

- Monitor urine output

- Monitor fluid input 
Table 1. Continued.

Question

Name three key indications to call a nephrologist for advice/review of a patient with AKI

\section{Predefined ideal answers}

One point each for:

- Refractory hyperkalaemia

- Refractory pulmonary oedema

- Refractory oliguria/anuria

- Any manifestation of uraemic syndrome

- Severe metabolic acidosis
Maximum score

$\mathrm{AKI}=$ acute kidney injury; $\mathrm{BP}=$ blood pressure; $\mathrm{CKD}=$ chronic kidney disease; MEWS = modified early warning system; JVP = jugular venous pressure.

Table 2. Scores broken down by question. Values are given as median [range].

\begin{tabular}{|c|c|c|c|c|c|c|}
\hline & $\begin{array}{l}\text { Definition } \\
(\max =3)\end{array}$ & $\begin{array}{l}\text { Categories } \\
(\max =3)\end{array}$ & $\begin{array}{l}\text { Risk factors } \\
(\max =5)\end{array}$ & $\begin{array}{c}\text { Examination } \\
(\max =3)\end{array}$ & $\begin{array}{l}\text { Observations } \\
(\max =3)\end{array}$ & $\begin{array}{c}\text { Renal referral } \\
(\max =3)\end{array}$ \\
\hline All trainees & $0.25[0-3]$ & $3[0-3]$ & $3[0-5]$ & $2[0-3]$ & $1[0-2.5]$ & $1[0-3]$ \\
\hline Foundation trainees & $0[0-3]$ & $3[0-3]$ & $3[0-5]$ & $1[0-3]$ & $1[0-2.5]$ & $1[0-3]$ \\
\hline Specialty trainees & $1[0-3]$ & $3[0-3]$ & $3[0-5]$ & $2[0-3]$ & $1.5[0-2.5]$ & $1[0-3]$ \\
\hline
\end{tabular}

\section{Results}

Questionnaires were completed by 146 trainees: 28 foundation and 27 specialty trainees from the trust hospitals and 53 foundation and 38 specialty trainees from the catchment hospitals. Of the 65 respondents of specialty grade, 52 were physicians.

The overall median score was 9.5 (range 0-17); this is broken down by grade in Fig 1. Foundation doctors did not perform as well as senior trainees (median score 8.5 (range 0-16.5) vs 10.5 $(2-17) ; \mathrm{p}=0.01)$. Among specialty trainees, there was no clear difference between physicians and surgeons, but numbers of the latter were small.

Table 2 gives scores according to the six key topics. Less than $2 \%(2 / 146)$ of trainees were able to define AKI according to the AKIN system, with 73 (50\%) unable to detail even one of its components (Table 1); however, 92 (63\%) categorised AKI correctly. Only $62(42 \%)$ trainees could name four or five risk factors for AKI, with $44(30 \%)$ managing two or fewer. Just $28(19 \%)$ trainees could provide three indications for renal referral, with $37 \%$ (54) unable to name even one such indication.

Fig 2 illustrates scores according to venue; no significant differences in performance were seen between trust and catchment trainees overall (median score $10.5(2-17)$ for trust trainees vs $9.5(0-16.5)$ for catchment trainees; $\mathrm{p}=0.4)$ or when broken down by grade (see Fig 3 a for foundation trainees $(p=0.18)$ and Fig $3 b$ for specialty trainees $(\mathrm{p}=0.69))$.

Fig 4 illustrates self-reported educational experience according to grade. Specialty trainees were far more likely to have received postgraduate teaching $(\mathrm{p}<0.0001)$, which reflects the limited postqualification experience of foundation trainees (2-18 months at the time of the survey). Conversely, foundation trainees reported receiving more undergraduate teaching $(\mathrm{p}=0.007)$. About $40 \%$ of trainees (56/142 respondents to this question) reported that they had never received any teaching on AKI.

Only 19/123 (15\%) respondents were aware of the NCEPOD report.

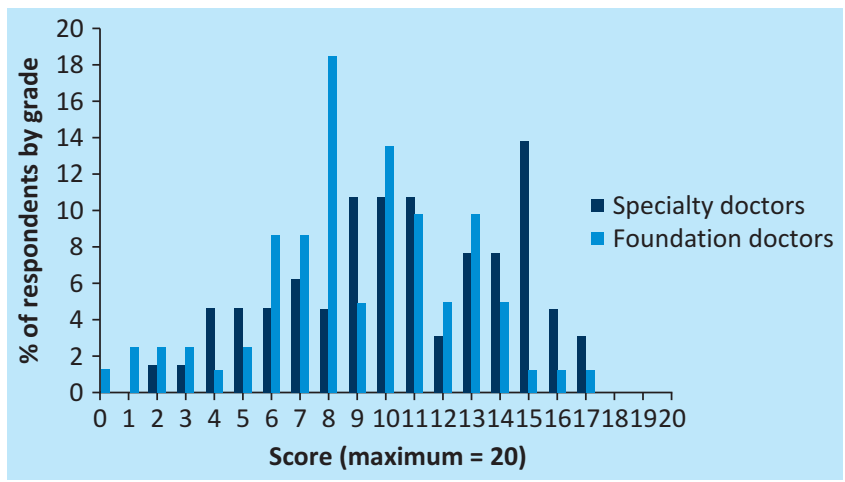

Fig 1. Questionnaire scores broken down by grade.

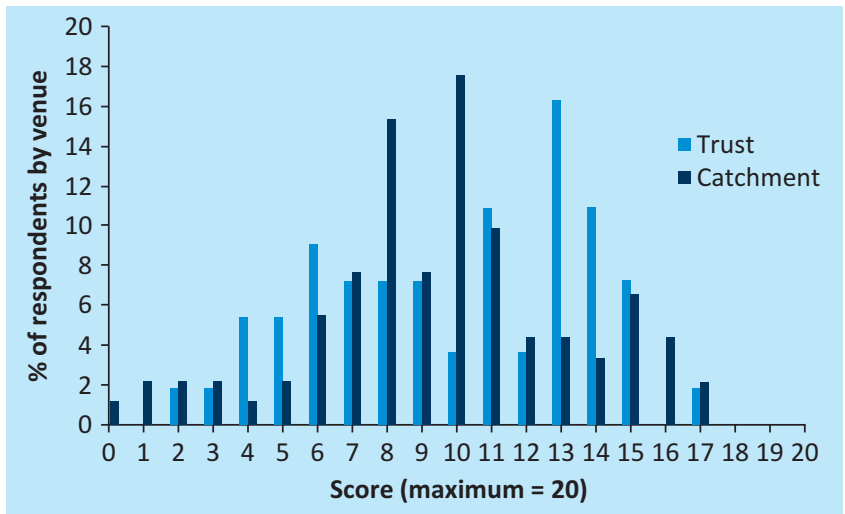

Fig 2. Questionnaire scores broken down by venue. 
Fig 3. Questionnaire scores broken down by venue for foundation (a) and specialty (b) trainees.
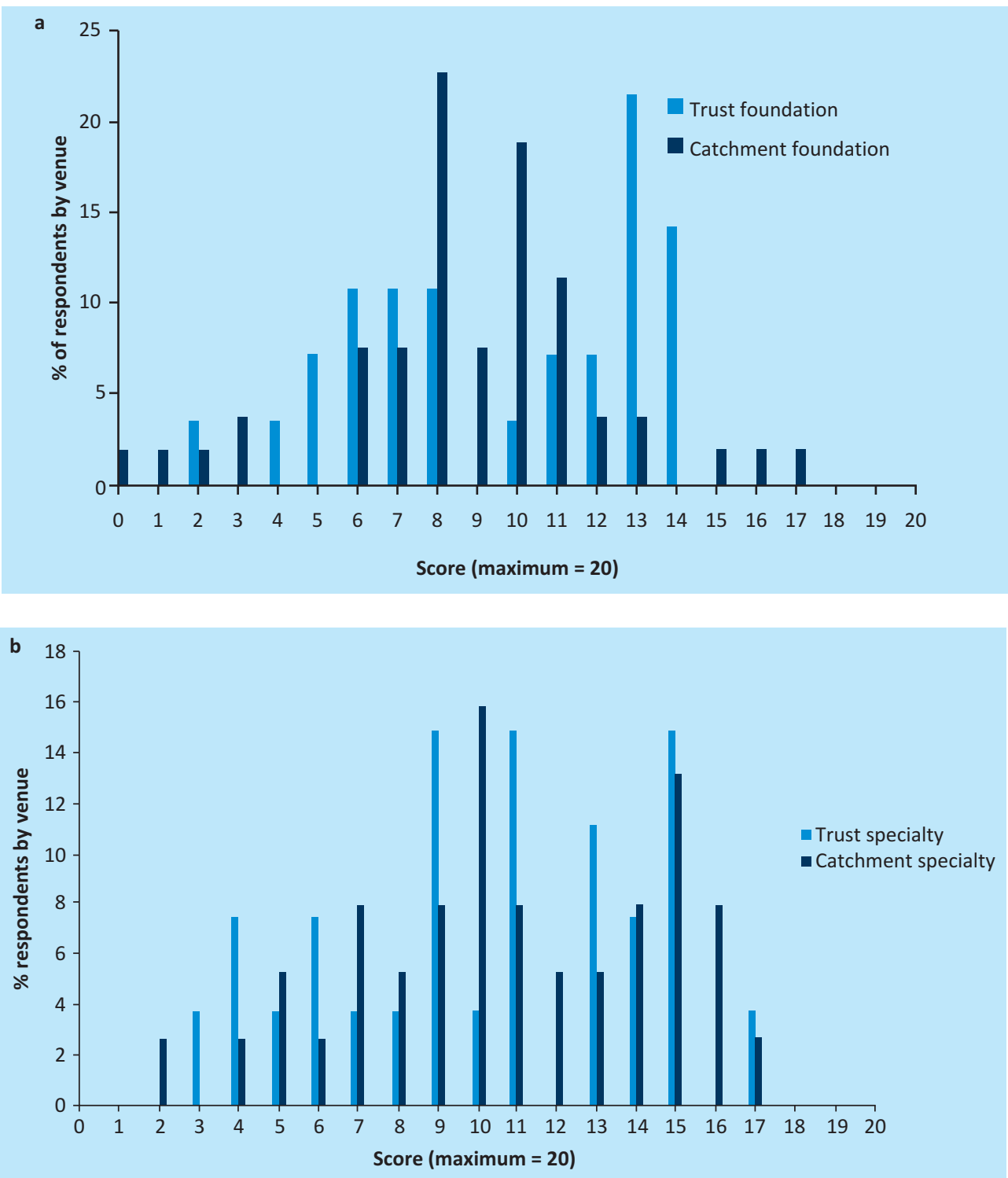

\section{Discussion}

Most AKI is caused by ischaemia, sepsis and/or nephrotoxicity ${ }^{18}$ and so has the potential to be prevented as long as 'at-risk' patients are recognised. Even when AKI has developed, its severity and duration may be ameliorated through prompt treatment of the initiating condition and avoidance of further insults.

Unfortunately, the NCEPOD report, ${ }^{13}$ which confirmed previous evidence, ${ }^{12}$ found that one-fifth of post-admission AKI was predictable and avoidable. Common findings included a failure to recognise unwell patients, infrequent blood monitoring and omissions in even basic fluid management. The NCEPOD report also found systematic delays in diagnosis and initial management, in part due to a straightforward failure to appreciate the significance of available data. Both NCEPOD ${ }^{13}$ and others ${ }^{12}$ reported delays in seeking specialist advice.

Routine nephrological input into the care of patients with AKI is not realistic - geography and limited resources prevent expert review in most such cases. At the heart of NCEPOD's recommendations, therefore, was a need to enhance non-specialist understanding and awareness of the prevention and management of AKI. However, the NCEPOD report was not clear on whether poor practice was due to gaps in basic knowledge or whether other, more insidious, problems might have been at play. As an example, a statutory reduction in working hours has led to an increase in shift work and may have disrupted the continuity of care previously engendered within traditional team-based practice. Furthermore, lack of attention to detail may not have been due to poor understanding but due to AKI being given a low priority amidst numerous other demands; the expectations of more senior, non-specialist clinicians might have compounded the problem.

Our educational survey was undertaken to assess the scale of any knowledge deficits among those clinicians most likely to be involved in the initial stages of preventing and managing AKI. Our results confirmed serious gaps in understanding of AKI. This was 
a

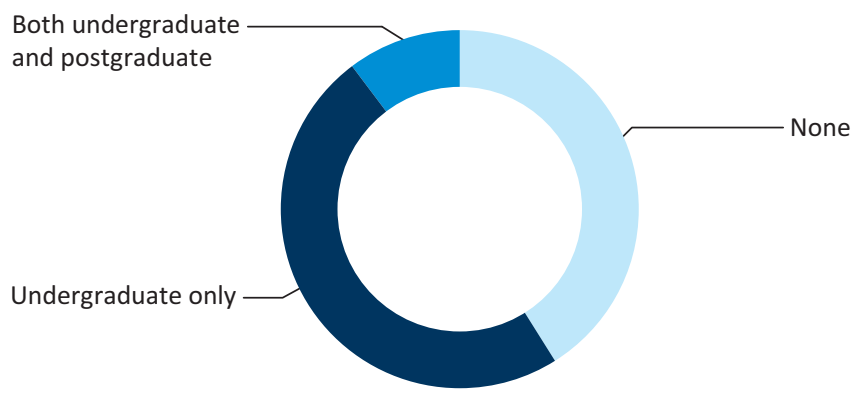

b

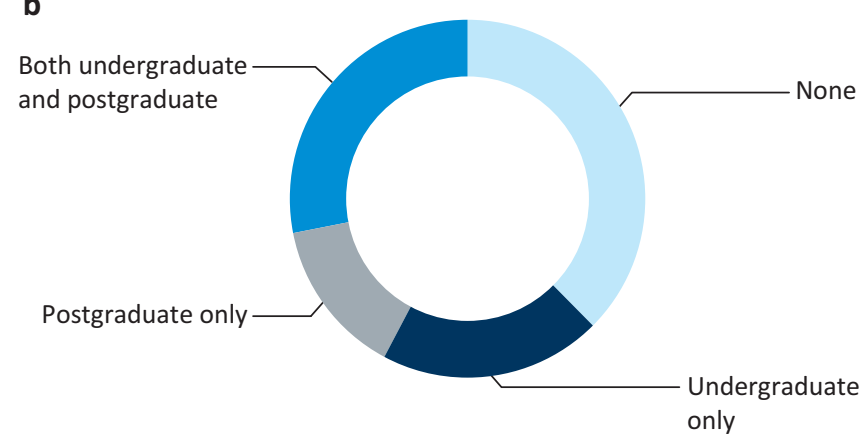

Fig 4. Self-reported teaching experience for foundation (a) and specialty (b) trainees.

evident across the grades; although, as might be anticipated, senior trainees scored higher. No differences were seen between trainees from the trust and the catchment area. As shown in Table 2, trainees scored highest when categorising AKI, but worrying deficiencies in this most basic of areas were still evident. In terms of educational experience, high proportions of trainees reported never having received any teaching on AKI. As might have been expected, specialty trainees were more likely to have received postgraduate teaching. Unexpectedly, significantly more specialty than foundation trainees reported that they had not had any undergraduate teaching on AKI. Clearly these apparent deficiencies in our teaching programmes are extremely concerning and warrant further examination.

It could have been argued that new terminology might have affected perceptions of actual educational experience. Arguing against this was the fact that most trainees were able to categorise AKI fully (median score = maximum score for that question; see Table 2), which suggests that there was no widespread confusion about nomenclature. In terms of actual teaching delivered, within the four years prior to the survey, we had given relevant talks to trust trainees on at least eight occasions and on one occasion for trainees at one of the catchment hospitals. Non-specialist trust trainees rotating through the renal unit (about 15 each year) also received teaching on AKI as part of their induction programme. An annual lecture on AKI is delivered to penultimate year undergraduates. In addition, all undergraduates receive teaching on recognition and initial management of the acutely unwell patient. The dichotomy between perceived and actual teaching experience highlights the pitfalls of self-reporting.
Our study also had other drawbacks. We did not collect data on self-directed learning, on the extent of any informal teaching or on the AKI component of 'non-trust' educational programmes. We also made an assumption that responses about teaching experience represented local provision, although most trainees do graduate locally. Our survey was not designed to assess organisational issues or to identify poor individual practice rather than poor understanding. Finally, and especially relevant to recent graduates, this snapshot survey was performed early in the annual academic cycle and so might not have captured teaching of AKI that was pending.

Addressing the identified knowledge deficits will require sustained educational efforts using a systematic approach that covers all key topic areas included in this survey. Furthermore, the questionnaire tool might be used as a template to quantify effectiveness. Organisational considerations include the priority and timing of educational sessions within already packed teaching programmes and how to free up extra time for those who might be expected to deliver these sessions. That these efforts might fail should at least be considered. Systematic aspects of working practice should be enhanced to target care of patients with AKI - for example, the detail of patient handover and the formalisation of risk assessment for AKI. Embedding care of patients with AKI within a regular, cross-organisational audit cycle might highlight areas of particular concern. Finally, innovative solutions, such as electronic clinical decision support systems, could be used to help protect patients from any remaining deficiencies in knowledge or practice.

\section{Conclusions}

All grades of junior medical staff surveyed demonstrate serious gaps in their knowledge of the basics of the prevention, diagnosis and management of patients with AKI. Our survey highlights the need for enhanced educational packages aimed at all training grades but also suggests that changes in organisation and infrastructure may be required to protect patients from these deficiencies.

\section{Acknowledgments}

We thank all of the trainees who kindly gave their time to complete the questionnaire and the programme organisers who allowed space within their meetings for this to be done.

\section{References}

1 Nash K, Hafeez A, Hou S. Hospital-acquired renal insufficiency. Am J Kidney Dis 2002;39:930-6.

2 Lassnigg A, Schmidlin D, Mouhieddine M et al. Minimal changes of serum creatinine predict prognosis in patients after cardiothoracic surgery: a prospective cohort study. J Am Soc Nephrol 2004;15:1597-605.

3 Uchino S, Bellomo R, Goldsmith D et al. An assessment of the RIFLE criteria for acute renal failure in hospitalized patients. Crit Care Med 2006;34:1913-7.

4 Kuitunen A, Vento A, Suojaranta-Ylinen R, Pettila V. Acute renal failure after cardiac surgery: evaluation of the RIFLE classification. Ann Thorac Surg 2006;81:542-6. 
5 Ahlstrom A, Kuitunen A, Peltonen S et al. Comparison of 2 acute renal failure severity scores to general scoring systems in the critically ill. Am J Kidney Dis 2006;48:262-8.

6 Mehta RL, Pascual MT, Soroko S et al. Spectrum of acute renal failure in the intensive care unit: the PICARD experience. Kidney Int 2004;66:1613-21.

7 Chertow GM, Burdick E, Honour M et al. Acute kidney injury, mortality, length of stay, and costs in hospitalized patients. J Am Soc Nephrol 2005;16:3365-70.

8 Ahlstrom A, Tallgren M, Peltonen S et al. Survival and quality of life of patients requiring acute renal replacement therapy. Intensive Care Med 2005;31:1222-8.

9 Schiffl H, Fischer R. Five-year outcomes of severe acute kidney injury requiring renal replacement therapy. Nephrol Dial Transplant 2008;23:2235-41.

10 Triverio P-A, Martin P-Y, Romand J et al. Long-term prognosis after acute kidney injury requiring renal replacement therapy. Nephrol Dial Transplant 2009;24:2186-9.

11 Hussein HK, Lewington AJP, Kanagasundaram NS. General management of acute kidney injury. Br J Hosp Med 2009;70:M104-7.

12 Stevens PE, Tamimi NA, Al-Hasani MK et al. Non-specialist management of acute renal failure. QJM 2001;94:533-40.

13 Stewart J, Findlay G, Smith N et al. Adding insult to injury: a review of the care of patients who died in hospital with a primary diagnosis of acute kidney injury (acute renal failure). London: National Confidential Enquiry into Patient Outcome and Death, 2009.

14 Hegarty J, Middleton RJ, Krebs M et al. Severe acute renal failure in adults: place of care, incidence and outcomes. QJM 2005;98:661-6.

15 Wright SE, Baudouin SV, Kaudeer N et al. Patient flow from critical care to renal services: a year-long survey in a critical care network. QJM 2008;101:643-8.

16 Mehta RL, Kellum JA, Shah SV et al. Acute Kidney Injury Network: report of an initiative to improve outcomes in acute kidney injury. Crit Care 2007;11:R31.

17 Lewington A, Kanagasundaram S. Clinical practice guidelines: acute kidney injury. Petersfield: Renal Association, 2011. www.renal.org/ Clinical/GuidelinesSection/AcuteKidneyInjury.aspx [accessed 28 March 2012].

18 Liano F, Junco E, Pascual J, Madero R, Verde E; Madrid Acute Renal Failure Study Group. The spectrum of acute renal failure in the intensive care unit compared with that seen in other settings. Kidney Int 1998;53(Suppl 66):S16-24.

Address for correspondence: Dr NS Kanagasundaram, Freeman Hospital, High Heaton, Newcastle upon Tyne NE7 7DN. Email: suren.kanagasundaram@nuth.nhs.uk

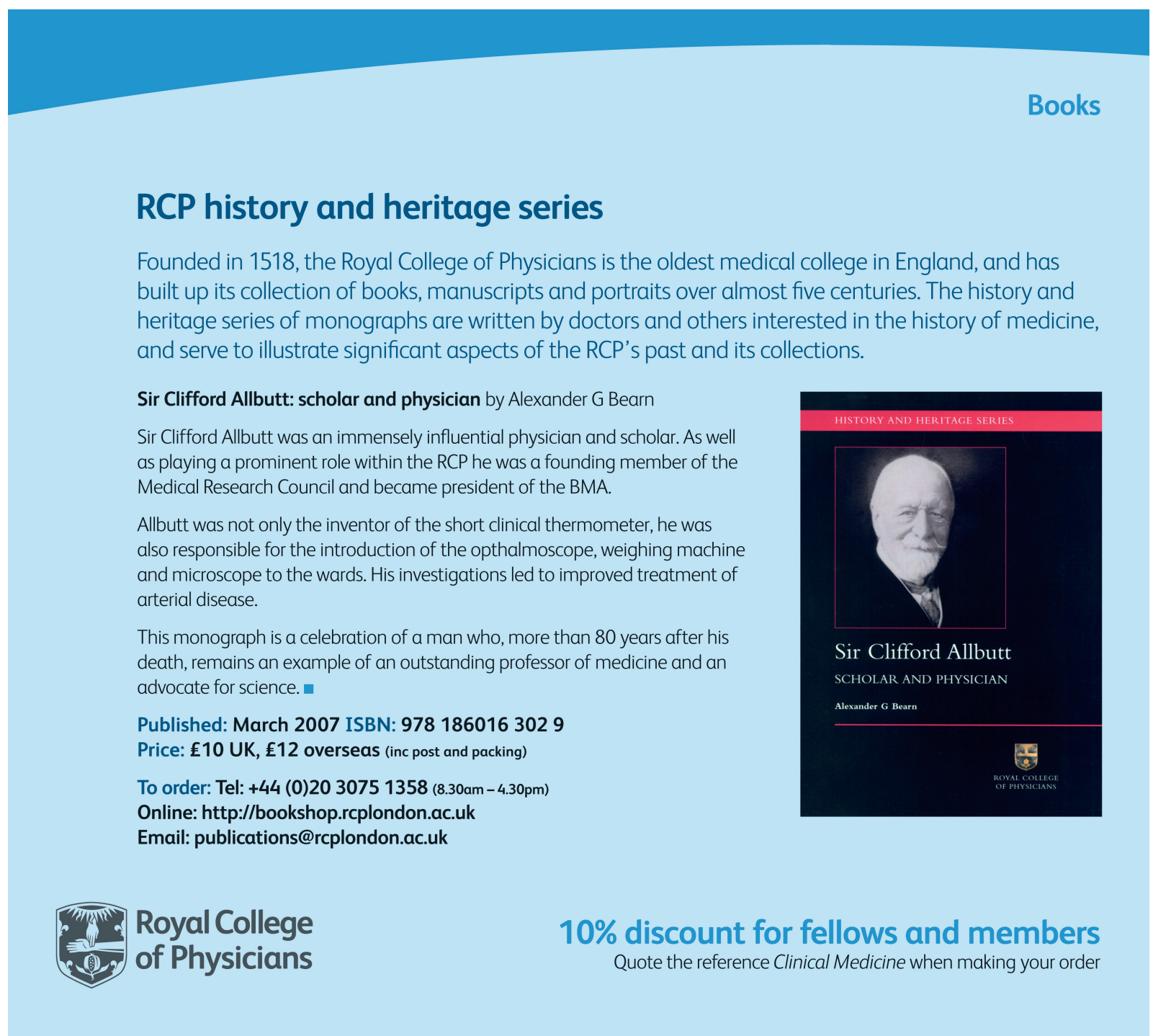

